\title{
A Novel Evolutionary Approach to Linear Time-Series Forecasting Model
}

\author{
Prakash Vijayan $^{1}$ and S. Suresh ${ }^{2}$ \\ ${ }^{1}$ Department of Mechanical Engineering, Indian Institute Of Technology, Madras \\ Chennai-600036, India, v_prakash81@yahoo.com \\ ${ }^{2}$ Department of Computer Science and Engineering, Indian Institute Of Technology, Madras \\ Chennai-600036, India, suresh_12may@yahoo.com
}

\begin{abstract}
This paper presents a handshake between the concepts of genetic algorithms and the forecasting problem to present a novel search based multiphase genetic algorithm to the forecasting problem based on the time series model. The backbone concept of the paper lies in utilizing the genetic approach effectively for implementing the Autoregressive process, a linear stochastic model where a time series is supposed to be a linear aggregation of random shocks. We propose to utilize the concept of genetic algorithms to transform an initial population of random suggested solutions to a population that contains solutions approximating the optimal one. A carefully chosen fitness function acts in the capacity of a yardstick to appraise the quality of each "chromosome" to aid the selection phase. We simulated the presented approach on a Pentium IV processor and obtained results that were very encouraging.
\end{abstract}

\section{Introduction and Previous Work}

Quantitative methods for generating forecasts of future outcomes using statistical procedures involve the examination of current and historically seasonally unadjusted data. This knowledge can be used to extrapolate the variable of interest. It is assumed that the process is stable over the forecast time horizon, but valid only for short time forecasts. Two types of quantitative forecasting models used are time series models and causal econometric models. Time series models involve a statistical analysis, which uses only historical data of the variable. Causal models are based on statistical analysis of data for other related variables and the use of these variables to forecast the variable of interest. There are several approaches for time series modeling. Some of them include Moving average, exponential smoothing and ARIMA, which are linear in nature. These numerous forecasting methods and the empirical findings, which are often in conflict, have given no clear guidelines as to the most appropriate methods for forecasting.

Box-Jenkins method [1] is popular because of its generality as it can handle any stationary or non-stationary time series, with or without seasonal elements. The BoxJenkins method for selecting an appropriate ARIMA model for estimating and forecasting a univariate time series consist of identification and testing and application. Although performance is primary concern, the choice of the Box-Jenkins 
model is due to its sound theoretical basis and the numerous research publications available. Box-Jenkins' ARIMA models are a class of linear models that were designed for linear time series. The model has been successfully applied in not only economic time series forecasting, but also for modeling the empirical dependencies between successive times between failures as discussed by Walls and Bendell [2]. The model gives satisfactory predictive performances as shown by Ho and Xie [3]. The model has been successfully used and tested for International tourist demand forecasting. This has been discussed in large and compared with different methods by Guerts and Ibrahim [4], Martin and Witt [5]. A typical problem in the ARIMA model for a time series is to estimate the coefficients of the Auto Regressive (AR) and the Moving Averages (MA) processes. Though, it is possible to fit a variety of linear models for a time series, parsimony is one important factor, which need to be considered. The number of terms used in the linear model should be a minimum. In the existing method, as demonstrated by Box Jenkins [1] in the identification stage, sample correlograms, the autocorrelation and partial autocorrelation coefficients are plotted to identify the order of the AR model. However, for higher order models, identification becomes tedious and difficult. An empirical investigation on the accuracies of the different forecasting methods has been discussed by Makridakis, S and Hibon, M[6]. The accuracy of a forecasting method is determined by analyzing the forecast error, which is defined as the actual value minus the forecast (or fitted) value of the variable for time period $\mathrm{t}$ :

$$
e_{t}=A_{t}-F_{t}
$$

where, $A_{t}$ the actual value at time $t ; F_{t}$ the forecast value at time $t+1$.

To the best of our knowledge, this is the first reported genetic approach to solve this problem. The field of genetic algorithms has a large amount of prior literature. Basics of genetic algorithms are presented in [9].

\section{Auto Regressive Process}

In the Autoregressive process, a linear stochastic model, a time series is supposed to be a linear aggregation of random shocks. However, parameter parsimony is an important criterion in choosing the right model. The stochastic process, as a linear model can be represented as

$$
\bar{Z}_{t}=e_{t}+\phi_{1} a_{t-1}+\phi_{2} a_{t-2}+\phi_{3} a_{t-3}+\phi_{4} a_{t-4}+\ldots \ldots
$$

The linear process represents $\bar{Z}_{t}$ as a weighted sum of present and past values of the white noise process $\mathrm{a}_{\mathrm{t}}$. The value $\bar{Z}_{t}$ is the deviation from the mean as the process is stationary and is represented by $\bar{Z}_{t}=\mathrm{Z}_{\mathrm{t}}-\mu$.

In the case, where the first ' $\mathrm{k}$ ' weights are non zero, the process becomes an Autoregressive process of order $k$, represented by AR(k) or ARIMA $(k, 0,0)$. The process can be written as,

$$
\bar{a}_{t}=e_{t}+\phi_{1} a_{t-1}+\phi_{2} a_{t-2}+\phi_{3} a_{t-3}+\ldots \ldots \ldots \phi_{k} a_{t-k}
$$


In the above process, the finite sets of weights $\phi_{1}, \phi_{2}, \phi_{3} \ldots . \phi_{k}$ are non-zero.

The first order Auto Regressive process or the Markov process can be represented as

$$
a_{t}=\mu+e_{t}+\phi_{1} a_{t-1}
$$

For stationarity of the process,

$$
-1<\phi_{1}<1
$$

Similarly, for a second order Auto regressive process, represented as

$$
a_{t}=\mu+e_{t}+\phi_{1} a_{t-1}+\phi_{2} a_{t-2}
$$

For stationarity of the process,

$$
-2<\phi_{1}<2 \text { and }-1<\phi_{2}<1
$$

The permissible values of $\phi_{1}$ and $\phi_{2}$ are for a stationary Autoregressive process as shown by Makridakis, Wheelwright and McGee[7], must fall in the triangle as shown in the figure 1.

\section{The Genetic Approach}

The algorithm presented in this paper apart from using only the genetic algorithm uses a search based multi-phase genetic algorithm that proceeds towards the optimal solution in an excellent manner. We start with a genetic algorithm by setting the ranges of the coefficients and operate in that range.

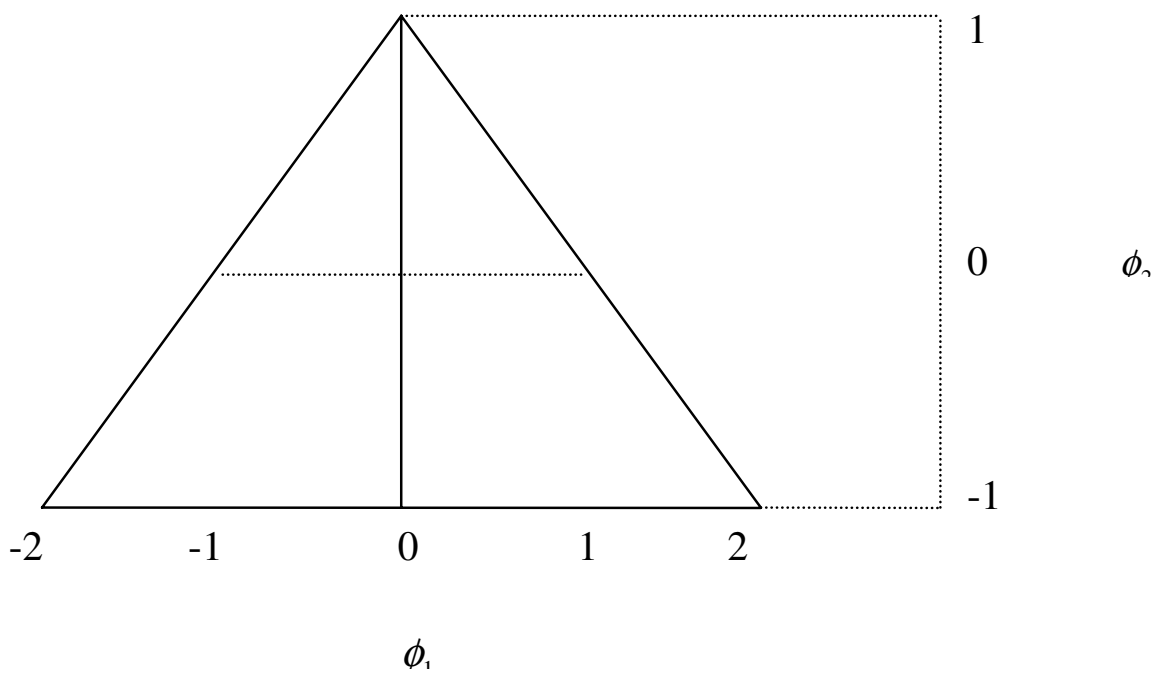

Fig. 1. The triangle shows the range of values that $\phi_{1}$ and $\phi_{2}$ for a stationary Autoregressive process. 
After simulating this genetic algorithm for a sufficiently large number of iterations we change the range of values that can be taken by the coefficients in the following manner:

- Observe the best ten solutions based on their fitness values.

- Obtain the maximum and minimum values taken by each of the coefficients and set them as the maximum and minimum values that can be taken by the respective coefficients during the next execution of the genetic algorithm.

This approach utilizes an intelligent and random methodology and therefore proceeds towards the optimal solution in an intelligent manner.

It is however essential to note that it is very important that we execute the first few genetic algorithms for sufficiently large number of iterations. The reason for such a claim is attributed to the fact that an improper prediction of range at the end of the first few executions may result in vast deviations of the solution from the optimal solution and therefore affecting the performance badly.

\subsection{Encoding}

The encoding phase essentially establishes a mapping over of the solution into a form that allows the genetic algorithm to analyze it. The encoding provided is an array named Coeff $[\mathrm{N}]$, where $\mathrm{N}$ represents the number of past values used for forecasting. Each element of the array consists of the coefficient taken by each of the past values in the forecasting equation. For example:

An array Coeff $[3]$ having the values $\{1.25,0.35,0.11)$ represents the equation of the form:

$$
a_{t}=1.25 a_{t-1}+0.35 a_{t-2}+0.11 a_{t-3}
$$

\subsection{Formation of the Initial Population}

The formation of the initial population determines the portion of the solution space explored by the genetic algorithm. Though the size of the initial population is up to the user, it should not be too small as it would greatly hinder the performance of the algorithm, while at the same time, a population too large in size would essentially increment the runtime as well since we would have to take care of quite a lot of chromosomes. Through extreme experimentation we found that an initial population of size equal to $10 \mathrm{~N}$, where $\mathrm{N}$ depicts the number of previous values used for forecasting gave us the best solution. We generate the initial population in a random manner but ensuring that the coefficients lie in the appropriate range. We generate $10 * \mathrm{~N}-3$ chromosomes using the random approach. The other three chromosomes are generated by setting all the coefficients in the array Coeff[N] to the maximum, minimum and the mid value of the ranges of the respective coefficients.

\subsection{Genetic Operators}

The genetic operators are applied to the population at hand to produce offspring that are, with a high probability, better than their parents. These operators ensure proper evolution of the current population towards the desired solution. 


\subsubsection{The Mating Operator}

For the mating process to take place, we would require the two parents to interact. The parents selected for mating are randomly chosen from the set of given population at hand. However the probability of selection of any chromosome is directly proportional to its fitness value. We adopt a one-point crossover strategy and the crossover point is selected randomly. The following example shows how mating is performed on two chromosomes:

Parent $1:\{1.5 \mid 0.5,0.12\}$

Parent2: $\{2.3 \mid 0.88,0.01\}$

When the above two parents are crossed over at the point shown by the 'l' they produce two offspring:

Child1: $\{1.5,0.88,0.01\}$

Child2: $\{2.3,0.5,0.12\}$

\subsubsection{The Mutation Operator}

The second genetic operator, the mutation operator, gives the genetic algorithm a greater touch of randomness. The randomness makes sure that no specific kind of pattern is lost at any point of time in the population. As a result of the mutation operator, we have the power to redirect an evolution that is converging to a local maximum, and place it on a path to a better solution. We assign a probability of mutation to each candidate of the population in a manner that ensures that a higher probability of mutation would be associated with those chromosomes that are of a low quality as far as the fitness value is concerned.

\subsubsection{Quality Enhancement Operator (QEO)}

This is the last genetic operator that we employed in an unconventional manner. This operator is primarily concerned with improving upon the quality of the chromosome on which it is operated. The QEO is employed on $1 / 4 *$ (Population size) number of randomly chosen chromosomes, the probability being same as the mutation probability. The operator functions in the following manner:

Calculate the value of the function:

$$
a_{t}=\text { Coeff }[0] a_{t-1}+\text { Coeff }[1] a_{t-2} \ldots . .+ \text { Coeff }[N] a_{t-N-1}
$$

If the value is more than the expected value for most of the given data, then randomly select any positive coefficient and decrement its value by $25 \%$ of its original value. It is important to note that by doing this, we ensure with a higher probability that the offspring produced is of a superior quality than its parent. This operator greatly enhances the efficiency of our algorithm.

\subsection{Fitness Value}

The sole purpose of the fitness function is to implement the "survival of the fittest" strategy. The fitness value in our case is solely concentrated on the minimization of deviation of the solution at hand from the actual solution. This optimization criterion is framed aptly in the form of a function that evaluates the chromosomes. The 
function Calculate fitness(Chromosome C) calculates the fitness value of the chromosome $\mathrm{C}$ in the following manner:

Calculate_fitness (Chromosome C)

Float MaxDev, CurrDev, Fitval

MaxDev = Maxdeviation()

For each series of data available begin

$$
\begin{aligned}
& \text { Calculate } a_{t}=\text { Coeff }[0] a_{t-1}+\text { Coeff }[1] a_{t-2} \ldots+\text { Coeff }[N] a_{t-N-1} \\
& \text { CurrDev }=\text { CurrDev }+\left(a_{t}-a_{t}^{*}\right)^{2} \text {, where } a_{t}^{*} \text { depicts the }
\end{aligned}
$$

desired value of $a_{t}$.

End for

Fitval $=1-$ CurrDev / (Maxdev* Number of series of data)

Return Fitval

End Function

The function Maxdeviation() calculates the square of maximum possible deviation of any chromosome which is calculated by substituting the upper bound values of each of the coefficients. Note that the series of data given can be formed by splitting the given series into smaller data series of requires length. For example:

In case of $\mathrm{N}=3$, a data series $1,2,3,4,5,6,7,8,9,10$ can be split into the following data series: $\{1,2,3,4\},\{2,3,4,5\},\{3,4,5,6\},\{4,5,6,7\},\{5,6,7,8\},\{6,7,8,9\}$ and $\{7,8,9,10\}$.

\subsection{The Main Algorithm}

The main algorithm of any phase of the genetic algorithm is as follows:

GA(Ranges of Coefficients)

Create initial population

while solution with the required fitness value has not been found do

Choose Parents for mating process

Pair up Parents Randomly for the mating process

Carry out Mating

Add Offspring to population

Carry out Mutation

Apply the QEO

End While

End Function

Note that, if at any point of time the population size exceeded the limit of 2000 chromosomes we filtered the population in the following manner: 
- Retain the top $50 \%$ of the chromosomes.

- From the bottom $50 \%$ of the chromosomes select $50 \%$ of them in random manner.

It was observed that such a policy adopted for controlling the population was highly lucrative.

\section{Experimental Section}

We implemented our algorithm in C language on a Pentium IV processor. We tested the working of our algorithm on the IBM common stock closing prices presented in [11]. We allowed our algorithm to proceed till the best solution presented by our algorithm remained stable for a significantly long time.

Before executing our algorithm we had to decide upon the various parameters of our genetic algorithm like the rate of mating, rate of mutation, size of initial population etc. By varying these parameters we were able to decide upon the best values for these parameters, which struck an optimal balance between the run-times, and the fitness values of the best solutions obtained.

The forecast equation obtained by our algorithm is:

$$
\nabla z_{t}=a_{t}+0.125( \pm 0.035) a_{t-1}-0.003( \pm 0.001) a_{t-2}
$$

The equation as predicted by the methodology proposed in [1] was:

$$
\nabla z_{t}=a_{t}+0.09( \pm 0.05) a_{t-1}
$$

It is very clear that the prediction of any value is mainly based on the previous two values as the coefficients associated with the remaining time lags are very close to zero. The residual variance of our algorithm is 43.45 , which is a significant improvement over the variance of 52.2 presented in [1]. Figure 2 shows the first differences obtained by our algorithm and the actual first differences. It is significant to note that our forecasts were very close to the desired values. The complexity of executing the algorithm was very less and the run-times were also impressive. Thereby our algorithm stood well on both the criteria as mentioned in section 1, as far as the forecasting accuracy and the ease of interpreting results is concerned.

\section{IBM daily stock closing price (Graph 4.3)}

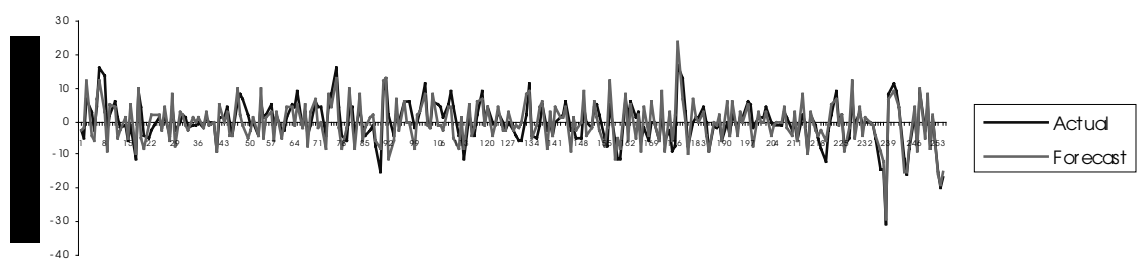

T ime

Fig. 2. The first differences obtained by our algorithm and the actual first differences 


\section{Conclusion}

The search based multi-phase genetic algorithm proposed in this paper has proved to be efficient in estimating the coefficients to very high accuracy. The results obtained were highly encouraging and the complexity associated with the execution was also very low. This paper opens up a whole new methodology for fixing the coefficients in the ARIMA model. Further research in this field may be oriented towards developing evolutionary approaches for identification and fixing the coefficients for the ARIMA model. There is also a tremendous scope of improvement in developing new crossover, mating and the fitness operators.

\section{References}

[1] Box GEP, \& Jenkins GM. Time series analysis: Forecasting and control, Revised ed. Holden-Day, San Francisco,1976.

[2] Walls, L. A., \& Bendall, A. (1987). Time series methods in reliability. Reliability Engineering, 18, 239-265.

[3] Ho, S. L., \& Xie, M. (1998). The use of ARIMA models for reliability forecasting and analysis. Computers and Industrial Engineering, 35

[4] Geurts, M.,\& Ibrahim, I. (1975). Comparing the Box-Jenkins approach with the exponentially smoothed forecasting model application to Hawaii tourists. Journal of Marketing Research,12, 182-188 .

[5] Martin, C. A., \& Witt, S. F. (1989). Forecasting tourism demand: A comparison of the accuracy of several quantitative methods. International Journal of Forecasting, 5,7-19.

[6] Makridakis, S., \& Hibon, M. (1979). Accuracy of forecasting: An empirical investigation. Journal of the Royal Statistical Society A, 142,97-145.

[7] Makridakis. S, Wheelwright SC, McGee VE, Forecasting: Methods and applications, John Wiley \& sons, 1983

[8] Kenneth A. De Jong and William M. Spears , "An Analysis of the Interacting Roles of Population Size and Crossover in Genetic Algorithms," In International Workshop Parallel Problem Solving from Nature, University of Dortmund, pages 38-47(1990).

[9] Kenneth A. De Jong and William M. Spears (1992), "A Formal Analysis of the Role of Multi-Point Crossover in Genetic Algorithms," In Annals of Mathematics and Artificial Intelligence Journal, v5, n1, pages 1-26.

[10] Pinaki Mazumder, Elizabeth Rudnick ,"Genetic Algorithms for VLSI Design, Layout and Test Automation ," Prentice Hall PTR, (2002).

[11] Box GEP, \& Jenkins GM. Time series analysis: Forecasting and control, $3^{\text {rd }}$ ed., Prentice Hall, pp. 542-543, IBM Common stock closing prices. 